\title{
Autosomal Dominant Gain-of-Function STAT1 Mutations in Bronchiectasis and Diabetes Mellitus
}

\author{
Ning $\mathrm{Xu}^{1 *}$, Zhuanbo Luo ${ }^{1}$, Yuefeng $\mathrm{Ye}^{2}$ and Fang Liu ${ }^{1}$ \\ ${ }^{1}$ Respiratory department of Ningbo No.1 Hospital, Ningbo city, China \\ ${ }^{2}$ Respiratory department of Cixi Longshan Hospital, Ningbo city, China
}

*Corresponding author: Ning Xu, Respiratory department of Ningbo No.1 Hospital, Ningbo city, Zhejiang province, China

\begin{abstract}
ARTICLE INFO
Received: 㠅 May 18, 2021

Published: May 27, 2021

Citation: Ning $\mathrm{Xu}$, Zhuanbo Luo, Yuefeng Ye, Fang Liu. Autosomal Dominant Gain-of-Function STAT1 Mutations in Bronchiectasis and Diabetes Mellitus. Biomed J Sci \& Tech Res 36(1)-2021. BJSTR. MS.ID.005801.
\end{abstract}

Keywords: Autosomal Dominant Gain-ofFunction STAT1 Mutation (AD-GOF STAT1 mutation); B lymphocyte (CD19+); Bronchiectasis; IgA; Type 1 Diabetes Mellitus (T1DM)

\section{ABSTRACT}

Background: Chronic mucocutaneous candidiasis and serious bronchiectasis are associated with autosomal dominant gain-of-function STAT1 mutations. But they were rarely reported accompany with diabetes.

Methods: A patient diagnosed with bronchiectasis, chronic mucocutaneous candidiasis and T1MD was investigated. Genetic analysis was performed as well as immunoglobulin assay and lymphocytic subtype analysis.

Result: The genetic analysis revealed a kind of AD-GOF mutation in STAT1 (nucleotide change c.1957G $>A$ and amino acid change p.V6531, locating in chr2-191841668 in exon 22). Besides, IgA decreased to $0.07 \mathrm{~g} / \mathrm{l}$ (normal range $0.8-2.3 \mathrm{~g} / \mathrm{l}$ ) in plasma and CD19+cells decreased to $0.5 \%$ (normal range 5-18\%) of lymphocyte in this case. The patient's symptom improved a lot after two weeks' hospitalization.

Conclusion: Decreased blood B lymphocytes (CD19+) and IgA levels might explain the susceptibility to chronic mucocutaneous candidiasis and bronchiectasis in patients with AD-GOF mutation in STAT1.

Abbreviations: AD-GOF: Autosomal Dominant Gain-of-Function Mutations; ANA: Antinuclear Antibodies; CRP: C-Reactive Protein; ESR: Erythrocyte Sedimentation Rate; GADA: Glutamic Acid Decarboxylase Negative Antibody; IAA: Insulin Autoantibody; ICA: Islet Cell Antibody; STAT: Signal Transducers and Activators of Transcription; T1DM: Type 1 Diabetes Mellitus

\section{Background}

Autosomal dominant gain-of-function mutation (AD-GOF mutation) in STAT1 were found to result in chronic mucocutaneous candidiasis and serious bronchiectasis [1,2]. Although some scattered cases have been reported, it is still a rare, complex and heterogeneous disease especially when complicated by other diagnosis. Recently, we treated a patient who experienced chronic mucocutaneous candidiasis, severe bronchiectasis and type 1 diabetes mellitus (T1DM). STAT1 heterozygous mutations, low level of blood B lymphocyte (CD19+) and IgA were detected.

\section{Case Report}

A 15-year-old female presented to our out-patient room with complaints of menstrual chronic cough? sputum production and diffuse bronchiectasis since early childhood (Figure 1). She had a history of T1DM for 3 years and underwent a long-term insulin replacement therapy. Family history suggested that her parents and her little brother were all healthy. The patient was admitted to our hospital for further treatment. Physical examination showed her mild fever of $37.8^{\circ} \mathrm{C}$, relative low oxygen saturation of $95 \%$ 
and rough moist rales in bilateral posterior lung fields. Red rashes with clear border and scurf scattered on her head, face and hands, as well as white spots on her oral mucosa (Figure 2). Laboratory tests on admission revealed a white cell count of $15,500 / \mathrm{mm} 3$ in which neutrophil dominated $\left(12,540 / \mathrm{mm}^{3}\right)$. Other plasmatic indicators including hemoglobin, platelet, c-reactive protein (CRP), erythrocyte sedimentation rate (ESR), fasting plasma glucose and $2 \mathrm{~h}$ postprandial glucose level was $122 \mathrm{~g} / \mathrm{l}, 120,000 / \mathrm{mm} \mathrm{3,43mg/l}$, $11 \mathrm{~mm} / \mathrm{h}, 23.73 \mathrm{mmol} / \mathrm{l}$ and $28.53 \mathrm{mmol} / \mathrm{l}$ respectively. Candida albicans grew in the sample of her pharyngeal swab, skin scraper and sputum cultures. We didn't find positive results in glutamic acid decarboxylase antibody (GADA), insulin autoantibody (IAA), islet cell antibody (ICA), HLA-B27 antigen, rheumatoid factor and antinuclear antibodies (ANA)in the subsequent test. But several results actually took our interest in the immunologic test and genetic analysis. In the immunologic test, the IgA level decreased to $0.07 \mathrm{~g} / \mathrm{l}$ (normal range $0.8-2.3 \mathrm{~g} / \mathrm{l}$ ) in her plasmatic sample while IgG, IgE, IgM, C3, C4 level were normal. The CD19+cells proportion also decreased to $0.5 \%$ (normal range $5-18 \%$ ) in lymphocyte subgroup analysis. In the genetic analysis, we detected nucleotide change c.1957G>A and amino acid change p.V6531, locating in chr2-191841668 in exon 22. But her parents and brother showed negative results in it (Figure 3), which implied her spontaneous mutation.

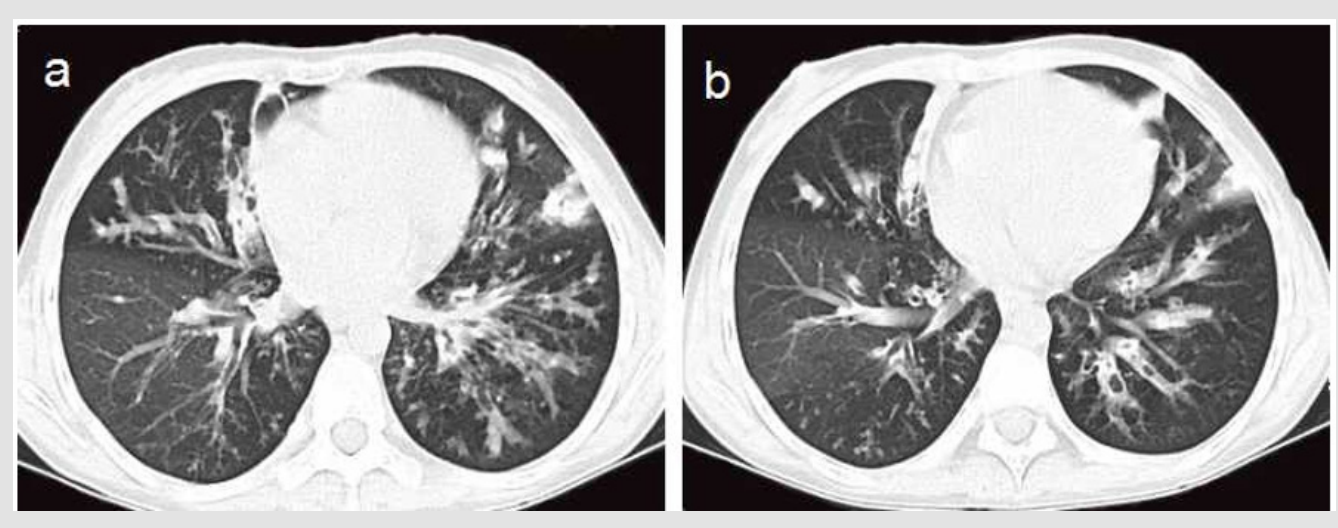

Figure 1: Computer tomography findings showing severe bronchiectasis and infection.
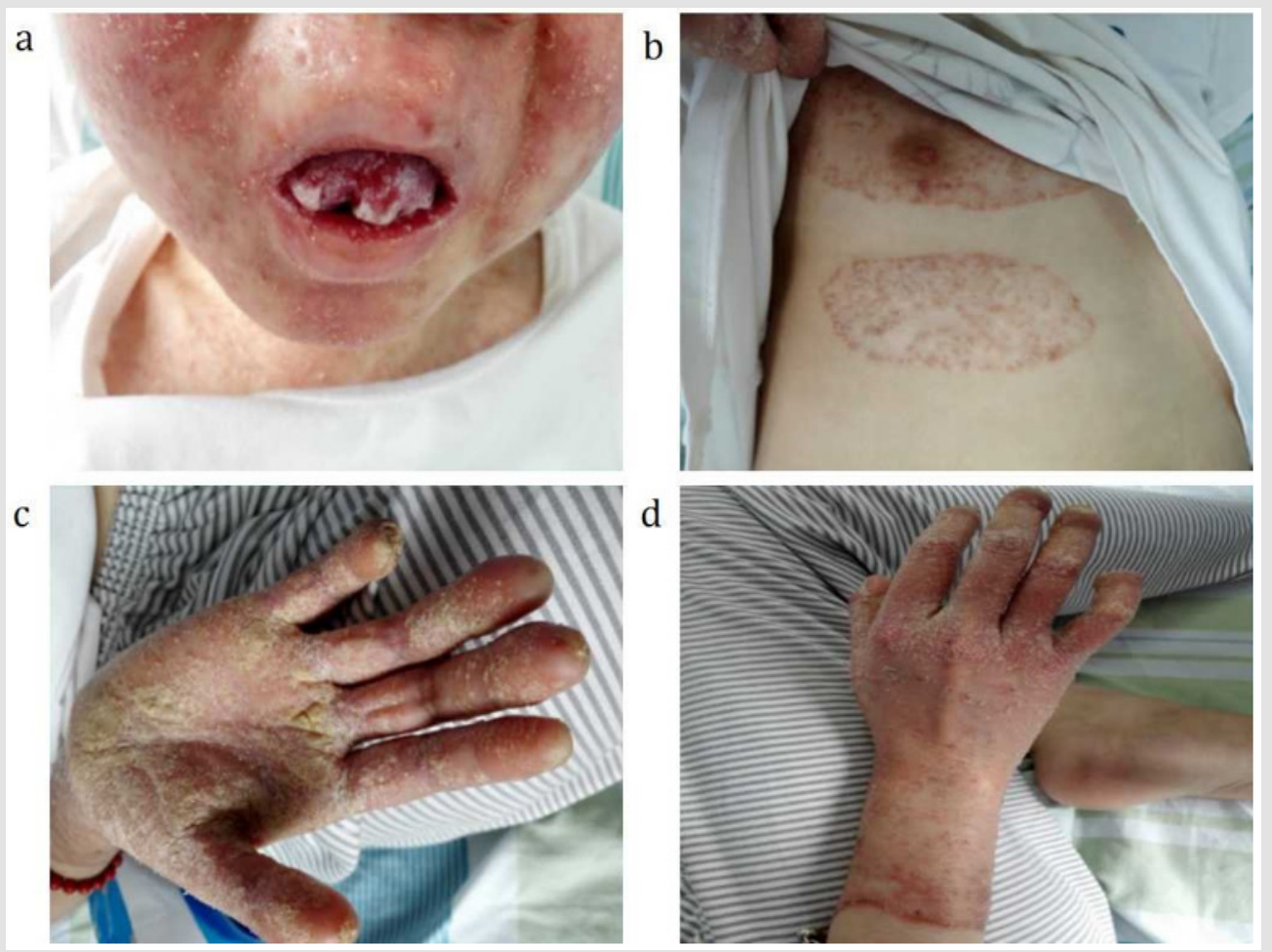

Figure 2: Genetic analysis report showing an autosomal dominant gain-of-function mutations (AD-GOF) in the signal transducer and activator of transcription 1 (STAT1) in this patient. 


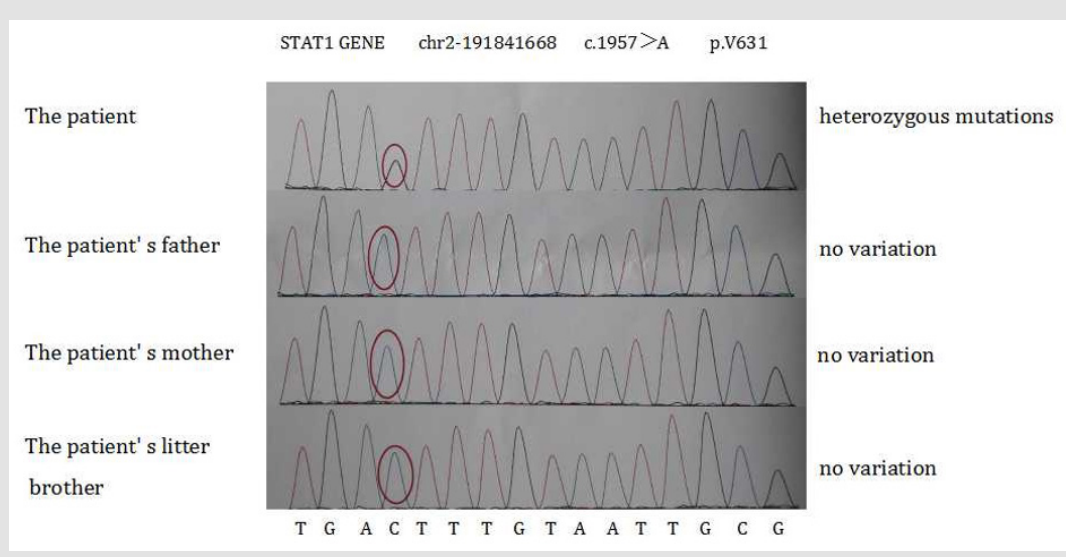

Figure 3: Skin and oral mucosal manifestations of our patient.

The patient was diagnosed with chronic mucocutaneous candidiasis accordingly after dermatologist consultation. We diagnosed bronchiectasis on the basis of HRCT results (Figure 1). We started on antibiotics including piperacillin-tazobactam and oral itraconazole in the setting of secondary infection of bronchiectasis as well as continue the insulin replacement therapy for T1DM. After two weeks' treatment, the symptoms improved significantly.

\section{Discussion and Conclusion}

Several studies found that STAT1 played an important role in inflammatory response and regulating apoptosis [3,4]. What's more, Liu et al. [5] described a case in which STAT1 heterozygous mutations caused chronic mucocutaneous candidiasis in 2001 although the pathogenesis of the disease was still in dispute. Van DE Veerdonk et al. [6] reported that STAT1 mutations could impair immune responses of type 1 and type $17 \mathrm{~T}$ helper cells (Th1 and Th17), which might lead to increased risk of fungal infection. It has also been implied that AD-GOF mutation in STAT1 may present diffuse bronchiectasis during early life possibly due to disease-associated B-cell dysfunction [2]. The STAT1 heterozygous mutations in our patient were accompanied with decreased blood B lymphocytes (CD19+) and IgA level. This might lead to poor immunity ability of skin, oral mucosa and bronchial mucosa, which would further cause chronic skin mucosal candidiasis and diffuse bronchiectasis. Type 1 diabetes antibodies (GADA, IAA, ICA) in her family were negative. The etiology and pathogenesis of diabetes were very complex and may also associated with gene and autoimmunity. But in this case, we failed to find positive T1MD associated antibodies (GADA, IAA, ICA) in her or her immediate family members and if there exist interactions between diabetes and the mutations we detected above.

Our case implied that decreased blood B lymphocytes (CD19+) and IgA levels might explain the susceptibility to chronic mucocutaneous candidiasis and bronchiectasis in patients with
AD-GOF mutation in STAT1. The interactions between diabetes and the mutations we detected (nucleotide change c.1957G $>$ A and amino acid change p.V6531, locating in chr2-191841668 in exon 22) needs further research.

\section{Acknowledgements}

None.

\section{Funding}

None.

\section{Availability of Data and Materials}

The data of this case can be found in the record system of Department of Respiratory Medicine, Ningbo First Hospital. Data are available from the corresponding author.

\section{Authors' Contributions}

Ning $\mathrm{Xu}$ wrote the initial manuscript. Zhuanbo Luo edited and submitted the manuscript. Yuefeng Ye, Fang Liu, CC were all involved in the diagnosis and treatment of the patient. All authors have read and approved the final manuscript.

\section{Ethics Approval and Consent to Participate}

None.

\section{Consent for Publication}

Written informed consent was obtained from the patient for publication of this case report and any accompanying images.

\section{Competing Interests}

None.

\section{References}

1. Boisson-Dupuis S, Kong XF, Okada S, Sophie Cypowyj, Anne Puel, et al. (2012) Inborn errors of human STAT1: allelic heterogeneity governs the diversity of immunological and infectious phenotypes. Curr Opin Immunol 24: 364-378. 
2. Oded B, Hagit D, Malena CC, Susanne Unger, David Shoseyov, et al. (2017) Autosomal dominant gain of function STAT1 mutation and severe bronchiectasis. Respir Med 126: 39-45.

3. De Prati AC, Ciampa AR, Cavalieri E, Raffaela Zaffini, Elena Darra, et al (2005) STAT1 as a new molecular target of anti-inflammatory treatment. Curr Med Chem 12: 1819-1828.

4. Kim HS, Lee MS (2007) STAT1 as a key modulator of cell death.Cell Signal 19: 454-465.

\section{ISSN: 2574-1241}

DOI: 10.26717/BJSTR.2021.36.005801

Ning Xu. Biomed J Sci \& Tech Res

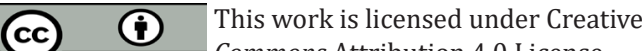

Submission Link: https://biomedres.us/submit-manuscript.php
5. Liu L, Okada S, Kong XF, et al. (2001) Gain-of-function human STAT1 mutations impair IL-17 immunity and underlie chronic mucocutaneous candidiasis. J Exp Med 208: 1635-1648.

6. Van de Veerdonk FL, Plantinga TS, Hoischen A Sanne P Smeekens, Leo AB Joosten, et al. (2011) STAT1 mutations in autosomal dominant chronic mucocutaneous candidiasis. N Engl Med 365: 54-61.

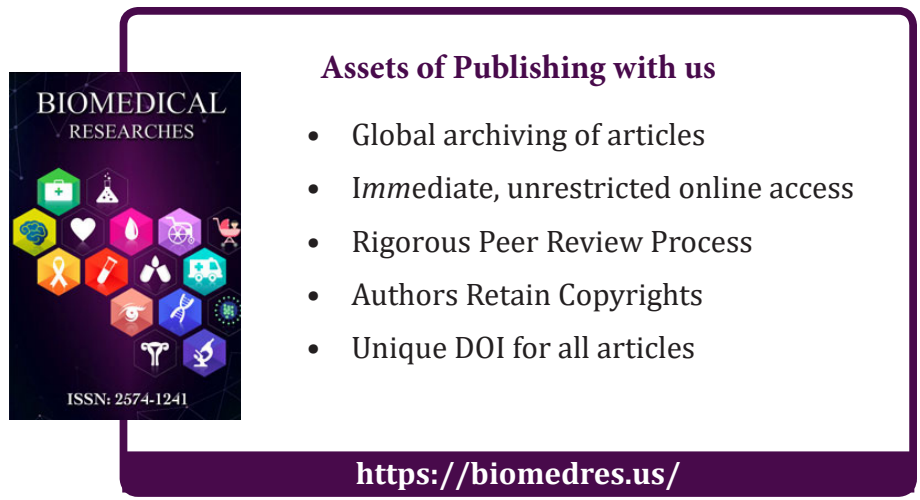

